Pub. Mat. UAB

no 10, Desembre 1978

UNA CONTRIBUCIO A L'ESTUDI DE GRUPS QUUE OPEREN SOBRE UN PRODUCTE

D'ESFERES.

M. Castellet ${ }^{\text {it }}$

\title{
1. Introduccio
}

L'estudi de grups finits que operen sense punts fixo: sobie una esfera esta íntimament ligat al dels grups periddics, es a dir grups per als quals la cohomologia és periddica o be, equivalentment, tals que liurs subgrups de sylow son clcilcs o quam. ternionics (1). En aquest treball considero grups finits que operen sense punts fixos sobre un prođucte $a^{\text {tesferes }} s^{n} \mathrm{xs}^{\mathrm{n}} i$ analitzo propietats homologiques i no,per tal que aixo sigui possible. La cohomologia utilitzada es sempre la de Tate i la no-: tacio la de (1) i (2).

2. Accio d'un grup finit sobre $s^{n} \times s^{n}$

$x$ sera. sempre una varietat compacta amb la mateixa, conomologia entera que $s^{n} \times s^{n}$; es a air $\mathrm{H}^{\circ} \mathrm{x}=\mathrm{z}, \mathrm{H}^{\mathrm{n}} \mathrm{x}=\mathrm{z} \oplus \mathrm{z}, \mathrm{H}^{2 n} \mathrm{x}=\mathrm{z}$.

* Aquest és un resurn d'un trebali realitzat l'any 1975 amb ajut d'una Borsa d'Estudis de la Societat Catalana de ciencies Fisiques, Quimiques i Matematiques. El trebali sencer sera publicat per l'Institut d'Estudis Catalans. 
$\mathrm{H}^{i} \mathrm{x}=0$ per $\mathrm{i} \neq 0 ; \mathrm{n}, 2 \mathrm{n}$.

Si $G$ es un grup finit que opera liiurament sobre $X$ podem considerar la successio espectral de Swan $\mathrm{E}_{1}^{\mathrm{p}, \mathrm{q}}$ associada a. I'acci6 de $G$ sobre $X(6)$; es te

$$
E_{2}^{P, Q}=H^{p}\left(G, H^{q} X\right)
$$

En virtud de les propietats cohomologigues de $X$ i tenint en compte que el bigrau de les diferencials $d_{i}$ es (i, 1-i) i que a l'operar G Iliurement, $E_{\infty}=0$, s obte una successio exacta liarga

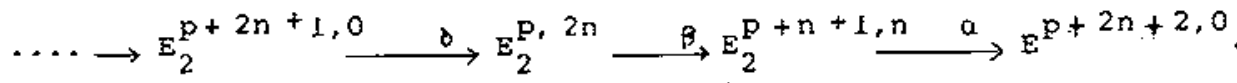

on 0.8 i $\partial$ poden ésser explicitades.

Suposen, ara, que l'accio de $G$ sobre $X$ conserva l'orientacio (es a dir, que el G-moduI $\mathrm{H}^{2 \mathrm{n}_{\mathrm{X}}}$ es trivial). Aleshores un

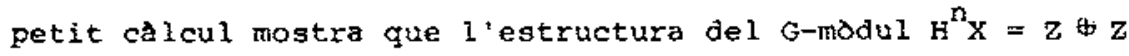
es tambe la trivial i per tant tenim:

Teorema Si $G$ és un grup finit que opera sense punts fixos i conservant I'orientacio sobre un producte d'esferes $s^{n} \times s^{n}$. es té una successio exacta llarga 


$$
\begin{aligned}
\cdots \rightarrow H^{p+2 n+1} G \stackrel{\partial}{\rightarrow} H^{P} G \stackrel{\beta}{\rightarrow} H^{p+n+1} G \in R^{p+n+1} G \stackrel{a}{\rightarrow} \\
\rightarrow H^{p+2 n+2} G \stackrel{\partial}{\rightarrow} \cdots \cdots
\end{aligned}
$$

Corol.lari En les condicions anteriors, si $|G|=r$ es te una successio exacta.

$$
\mathrm{O} \rightarrow \mathrm{H}^{\mathrm{n}} \mathrm{G} \rightarrow \mathrm{H}^{\mathrm{n}+1} \mathrm{G} \rightarrow \mathrm{Z} / \mathrm{rZ} \oplus \mathrm{Z} / \mathrm{rZ} \rightarrow \mathrm{H}^{\mathrm{n}+\mathrm{I}} \mathrm{G} \rightarrow \mathrm{H}^{\mathrm{n}} \mathrm{G} \rightarrow \mathrm{O}
$$

(Ja que la finitud de $G$ ens dona I'isomorfisme $\mathrm{H}^{\mathrm{n}} \mathrm{G}=$ $=$ Hom $\left(\mathrm{H}^{-n} \mathrm{G}, \mathrm{Z} / \mathrm{ZZ}\right)=\mathrm{H}^{-\mathrm{n}} \mathrm{G}$.

\section{Grups cohomoldgicament semiperiodics}

Diré que un grup finit $G$ d'ordre $r$ es cohomologicament semiperiddicon (c.s.p.-n) si existeix una successio exacta $\mathrm{O} \rightarrow \mathrm{H}^{\mathrm{n}-1} \mathrm{G} \rightarrow \mathrm{H}^{\mathrm{n}} \mathrm{G} \rightarrow \mathrm{Z} / \mathrm{rZ} \oplus \mathrm{Z} / \mathrm{rZ} \rightarrow \mathrm{H}^{\mathrm{n}} \mathrm{G} \rightarrow \mathrm{H}^{\mathrm{n}-1} \mathrm{G} \rightarrow \mathrm{O}$

Proposicio Tot grup periddic-n és c.s.p.-n

Proposicio Si G Es c.s.p.-n, aleshores

$$
\mathrm{H}^{\mathrm{n}} \mathrm{G} / \mathrm{H}^{\mathrm{n}-1} \mathrm{G}=\mathrm{z} / \mathrm{hz} \in \mathrm{z} / \mathrm{kz} \text { on } \mathrm{h}, \mathrm{k}=\mid \mathrm{Gl}
$$

(És conseqüencia de la definicio i del teorema de Kulikov (5))

Proposicio El producte directe d'un grup periodic-n per un grup periddic-m és c.s.p.-(m,c.m. (n,m)).

(Consequancia de les formules de künneth). 
Corol.Iari Si Qs es el grup de quaternions generalitzat d'ordre s, es te
a) $z / r z \oplus z / s z$ ss c.s.p. -2
b) $\mathrm{Z} / \mathrm{rZ} \times \mathrm{Q}_{\mathrm{s}}$ és c.s.p. -4
c) $Q_{I} \times Q_{s}$ es c.s.p. -4

Teorema $G=z / p Z \oplus z / p Z \oplus z / p Z$ no es c.s.p. $-n$ per a cap $n$. Demostracio: Com que $H^{2 n}{ }_{G}=G i H^{2 n-1} G=0$, no pot existir cap successio exacta com la del corol.lari de I'apartat 2, ja que a $G$ hi ha $p^{3}-1$ elements $d$ 'ordre $p$ i a $z / p^{3} z \oplus z / p^{3} z$ nomes n'hi ha $p^{2}-1$.

Corol.lari $z / p Z \oplus z / p Z \oplus z / p Z$ no pot operar sense punts fixos i conservant 1'orientacio sobre cap producte d'esferes de la mateixa dimensi6.

Aquest corol.lari ja fou demostrat per Heller l'any 1959 amb metodes totalment diferents (3). La demostracio que $n$ 'he donat te dos avantatges: dona un resultat algebraic més fort i es força més curta i elegant.

\section{Grups semiperiodics}

El teorema d'Artin i Tate que he mencionat a la introduccio aona una caracteritzacio dels grups periddics en termes $\sim 94$ 
de teoria de grups classica: tot subgrup de sylow ha d'ésser clclic o quaternionic. Es planteja, aleshores, la possibilitat d'obtenir una caracteritzacio semblant per als grups c.s.p.

Dire que un grup $\mathrm{g}$ es semiperiodic si tot subgrup de sylow de $G$ és un producte de dos grups cf́clics o quaternionics.

\section{Proposicio}

a) Tot grup periddic es semiperiodic

b) El producte directe de dos grups periodics es semiperiodic.

c) Tot grup abelia semiperiddic és la suma directa de dos grups ciclics.

Corol.lari Tot grup abelia semiperiddic és c.s.p.

El resultat següent permet determinar quins grups nilpotents son semiperiðdics.

Teorema Sigui $G$ un grup finit nilpotent semiperiddic. Aleshores:

a) $|\mathrm{a}|=2^{a+b} \cdot q^{c+d},(2, q)=1, a+b \geq 3, a \leq b, c \leq d$.

$$
\mathrm{G}=\mathrm{s}_{2} \mathrm{G} \times\left(\mathrm{z} / \mathrm{q}^{\mathrm{c}} \mathrm{z} \times \mathrm{z} / \mathrm{q}^{\mathrm{d}} \mathrm{z}\right) \text { on } \mathrm{s}_{2} \mathrm{G}=\mathrm{z} / \mathrm{2}^{\mathrm{a}} \mathrm{z} \times \mathrm{Q}_{2} \mathrm{~b} .
$$$$
\mathrm{Q}_{2} \mathrm{a} \times \mathrm{z} / \mathrm{2}^{\mathrm{b}} \mathrm{z} \quad \mathrm{O} \quad \mathrm{Q}_{2} \mathrm{a} \times \mathrm{Q}_{2} \mathrm{~b}
$$ 
b) G esc.s.p. -4 i

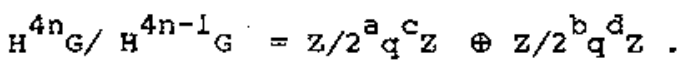

\section{Alqunes propietats d'extensio}

Els resultats d'aquests apartats estan encaminats a demostrar $1^{t}$ equivalencia entre semiperiodic i c.s.p. o si mes no a sugerir com $\mathrm{s}^{\text {tha }}$ de modificar el concepte de c.s.p. per tal de que valgui l'equivalencia.

Proposicio El producte semidirecte $\mathrm{z} / \mathrm{rZ} \mathrm{z} / \mathrm{sZ}$ de dos grupg clalics es c.s.p. $-(2 \mathrm{~m})$ on $m$ es l'enter positiu mes petit tal que $t^{m} \equiv 1$ mod $r$, essent $t$ la imatge de $1 \in \mathrm{z} / \mathrm{rz}$ per 1 'accio de $1 \in \mathrm{z} / \mathrm{sz}$.

(La demostracio utilitza els calculs fets per wall a (7)).

-. Corol.lari $\mathrm{z} / \mathrm{rz}$ i $\mathrm{z} / \mathrm{sz}$ nomes pot operar sense punts fixos

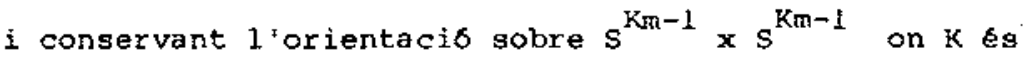
parell i m com a la proposicio anterior.

Proposicio Sigui $G$ una extensio d'un grup periodic $Q$ per un grup ciclic $N=z / r z$. Si $2 m$ no es un periode de $Q$ o si $N^{2} \neq N$, el grup $G$ no es c.s.p. $-(2 m) i$, per tant, no pot 
operar sense punts fixos i conservant litorientacis sobre $s^{2 m-1} \times s^{2 m-1}$

La demostracio resulta del fet que

$$
\mathrm{H}^{\mathrm{n}} \mathrm{G}=\mathrm{H}^{\mathrm{n}} \mathrm{Q} \oplus \mathrm{H}^{\dot{0}}\left(\mathrm{Q}, \mathrm{H}^{\mathrm{n}} \mathrm{N}\right)
$$

consequencia de l'estudi de les diferencials de la successio espectral de Lyndon-Hochschild -Serre (4).

El punt essencial en la demostracio de la possible equivalència entre semiperiodic $i$ c.s.p. radica en el fet que tot subgrup d'un grup c.s.p. sigui o no c.s.p. La següent proposicio $n^{7}$ es un resultat parcial.

Proposicio si G és c.s.p.-n i $U=s_{P} G$ és abelia, $U$ es c.s.p. -n. 


\section{BI BLIOGRAFIA}

(1) H. Cartan- S.Eilenberg "Homological Á Igebra" Princeton 1956

(2) M. Casteliet "Grupos finitos con cohomología pexiodica $y$ espacios que admiten recubrimientos esfericos" Tesis doctoral. Barcelona 1973

(3) A. Heller "A note on spaces with operators" Illinois J. Math. 3(1959), 98-100

(4) P.J. Hilton- U.Stambach "A course in homological Algebra" Grad. Tetx in Math.4 Springer 1971

(5) A. Kurosh "The theory of groups" Part I Chelsea Pub.

(6) R. Swan "A new method in fixed point theory" Comment. Math. Helv, 34(1960), 1-16

(7) C.T.C.Wall "Resolutions for extensions of groups" Proc. Cambriage Phil.soc. 57 (1961), 251-255 